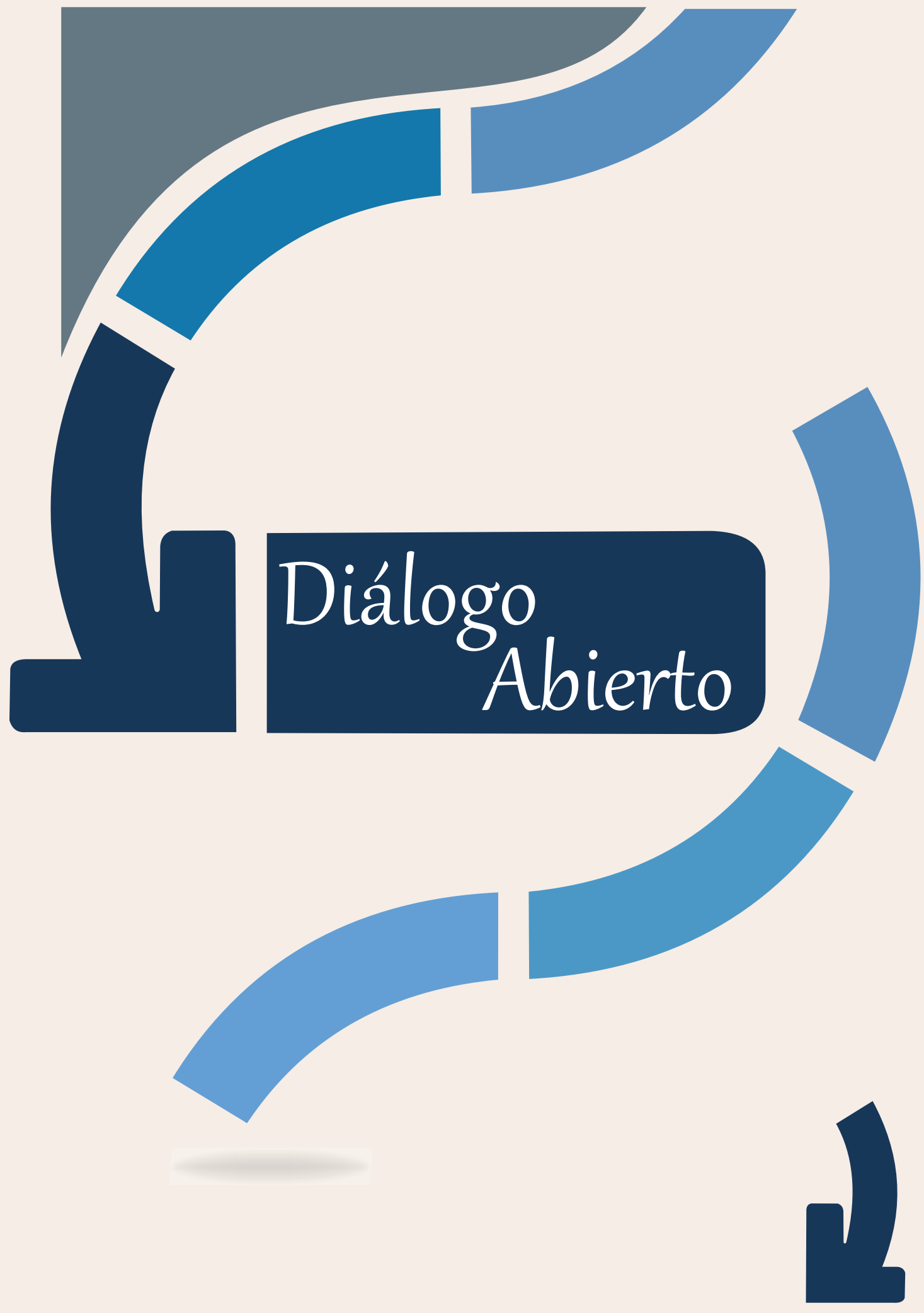





\section{Capacitación sobre planeación y evaluación efectivas de proyectos}

Astarte Alegría Castellanos ${ }^{1}$

Perla Simons Morales ${ }^{2}$

\section{CTI ¿Cómo contribuye la UIC a la formación de profesores en el área de la salud?}

AA/La UIC es una Unidad Académica líder en la Facultad de Ciencias Médicas en materia de gestión académica de la investigación en salud, como tal entre sus funciones está la de capacitar a los docentes en investigación, particularmente en el área de la salud.

PSI La UIC en la Facultad de Ciencias Médicas, como uno de sus objetivos, pretende brindar las herramientas a los profesores y estudiantes de grado \& posgrado para que puedan desarrollar la investigación científica en las mejores condiciones. Como resultado, surgen los talleres de Planeación y Evaluación Efectivas de Proyectos (PEEP), los cuales se desarrollan desde año 2008 en colaboración con aliados estratégicos como el Programa Especial de Entrenamiento e Investigacion en Enfermedades Tropicales (TDR) de la Organización Mundial de la Salud (OMS), Ginebra, Suiza; la Organización Panamericana de la Salud (OPS); y el Centro Internacional de Entrenamiento e Investigaciones Médicas (CIDEIM), Cali, Colombia.

\section{$\mathrm{CTI}$ ¿Qué significan los cursos PEEP? ¿A quién va dirigido el curso? ¿Qué metodología utilizan? ¿Cuál es la duración del curso?}

AAl Los cursos de Planeación y Evaluación Efectivas de Proyectos, conocidos por sus siglas como Cursos PEEP, son una capacitación ofrecida por la UIC a la comunidad docente de la FCM. Esta oferta es, a la vez resultado de una gestión internacional de esta Unidad.

\footnotetext{
${ }^{1}$ Psic, MSP. Coordinadora del Eje de Ciencias Sociales y profesora de la Facultad de Ciencias Médicas, Universidad Nacional Autónoma de Honduras, Tegucigalpa, Honduras. Contacto: astarte.alegria@unah.edu.hn

2 Enf, MSP. Coordinadora de la Unidad de Investigación Científica, Facultad de Ciencias Médicas, Universidad Nacional Autónoma de Honduras, Tegucigalpa, Honduras. Contacto: perla.morales@unah.edu.hn
} 
Se trata esencialmente de un curso de habilidades, es decir, que complementa, perfecciona, desarrolla capacidades para planear, organizar, gestionar y evaluar proyectos de investigación. Se caracteriza por una metodología de aprendizaje activa, de interacción entre participantes y con grupos de trabajo, lo que se denomina "aprender haciendo", metodología que indudablemente facilita el aprendizaje de los participantes. Otra característica es su breve duración, ya que los contenidos organizados a través de módulos, los cuales están excelentemente ajustados.

PSI Los Cursos PEEP se imparten a profesionales de la salud: profesores, miembros de Secretaria de Salud, doctores nacionales e internacionales. (Guatemala, El Salvador y Costa Rica). Son cursos de 28 horas presenciales y varias horas de trabajo individual (durante 4 días) en los cuales se forman 4 grupos de 3 personas para desarrollar ejercicios en conjunto. La estrategia pedagógica está bien diseñada. Son dos tipos de cursos taller: Habilidades Básicas y Capacitador de Capacitadores.

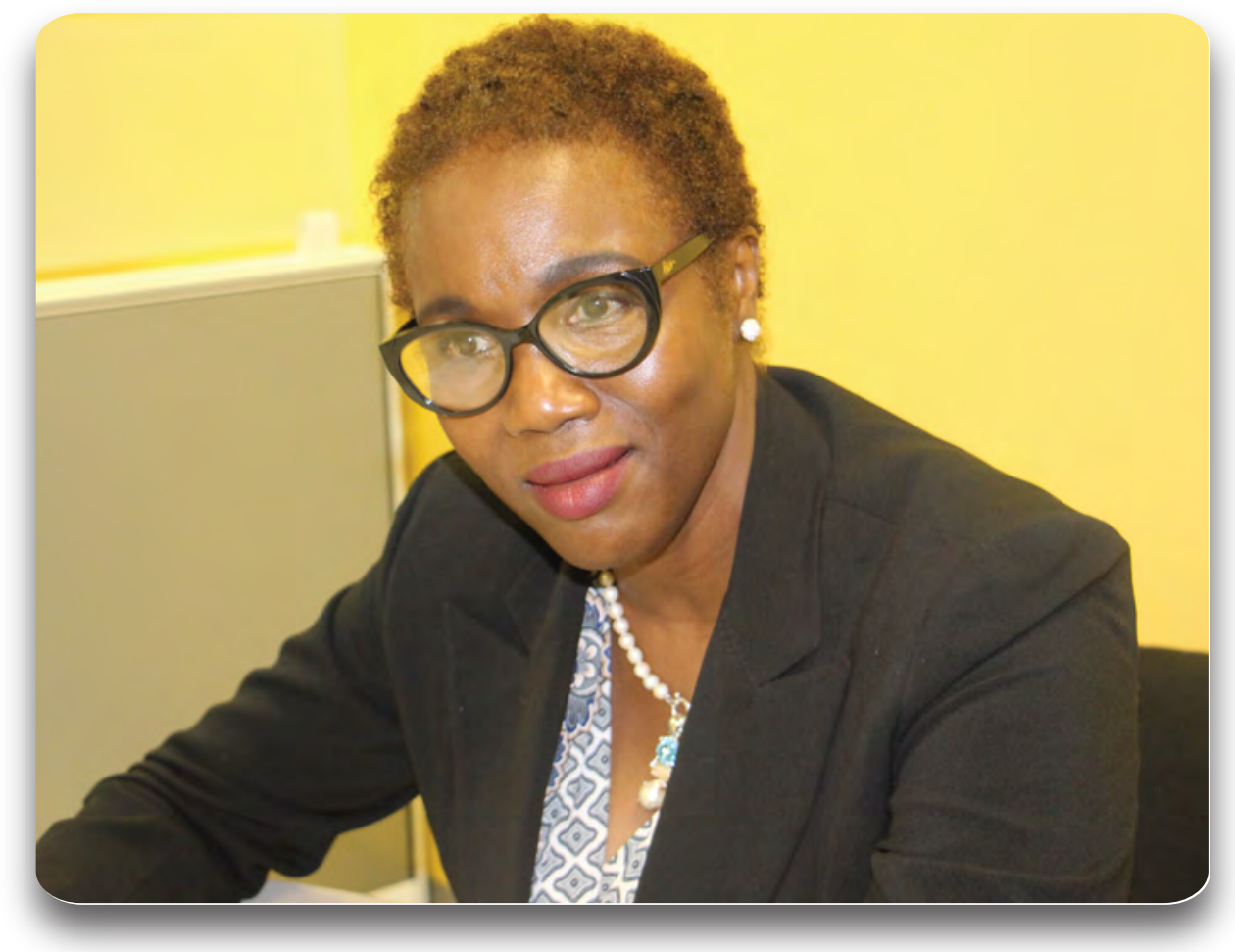

Perla Simons Morales 


\section{CTI ¿Qué habilidades desarrollará el participante al finalizar el curso?}

AA/Partamos de que el participante tiene una idea para un proyecto de investigación. EI PEEP le dotara de habilidades (capacidades) para planear y organizar este proyecto de investigación; en otras palabras, le dará a esta idea la forma de un proyecto de investigación partiendo de la definición del problema, sus objetivos y metas a través de plan de trabajo para alcanzar los objetivos establecidos. Todo ello, sustentado en teorías y conceptos, complementados con herramientas de planificación

PSI El Curso de Habilidades Básicas, se centra en objetivos para realizar un proyecto 0 una investigación. Inicia con una parte introductoria para poder delimitar esos objetivos y los elementos que se plantean en un proyecto de investigación 0 proyecto de gestión. Los participantes aprenden a programar las actividades de su proyecto, utilizando el programa Microsoft Project, uso de gráficas de GANTT, entre otros.. Luego, ellos establecen indicadores de medición de resultados.

El Taller de Capacitación de Capacitadores se imparte después del taller de habilidades básicas, siempre y cuando el participante manifieste su interés. El participante se compromete a devolver lo que se le dio en el taller inicial, cuando él se convierte en capacitador. Se hace un entrenamiento práctico de cómo capacitar a otros profesionales sobre competencias básicas de planificación y evaluación efectiva de proyectos. Metodología de los cursos: "aprender haciendo". El material educativo ya está diseñado previamente, de manera estandarizada. La UIC se encarga de reproducir el material.

\section{CT/¿Cuál es el proceso de selección de los participantes?}

AA/La disponibilidad e interés del docente. Desde lo formal, se requiere un perfil (idea) de lo que será el proyecto de investigación en salud, dominio básico de computación. Considero que el apoyo y colaboración de los departamentos de los cuales provenga el participante es importante para que el PEEP como herramienta incida en desarrollo de capacidades no solo como departamento, sino como Facultad. Es importante señalar que el PEEP se ha adaptado como un Módulo de Gestión de la Investigación en el programa académico de la Maestría de Salud Pública, desarrollado en el primer o segundo semestre.

PSI El proceso de selección se realiza anunciando a los posibles interesados en el área de la salud. Debido a los cupos limitados, se envían cartas a los jefes de 
departamento para que proponga 2 candidatos. En el caso de participantes internacionales, trabajamos en conjunto con CIDEIM y la OPS para que ellos puedan comprar los pasajes y encargarse del alojamiento; nosotros nos encargamos de seleccionar el local apropiado para los talleres y ofrecer la logística local (los coffee breaks, copias, insumos oficina).

\section{CT/¿Cuándo será la próxima capacitación de PEEP?}

AA/En diciembre próximo, año 2018, se llevara a cabo el curso de capacitación de capacitadores.

PSI Tenemos previsto un siguiente taller de Capacitación de capacitadores para el mes de diciembre, año 2018, en donde participaran 2 capacitadores del área de enfermería y 2 capacitadores de la UIC, con apoyo de una capacitador internacional de CIDEIM. Asimismo, ahora con mayor recursos de capacitadores, anunciamos que ya estamos planificando los cursos PEEP del año 2019.

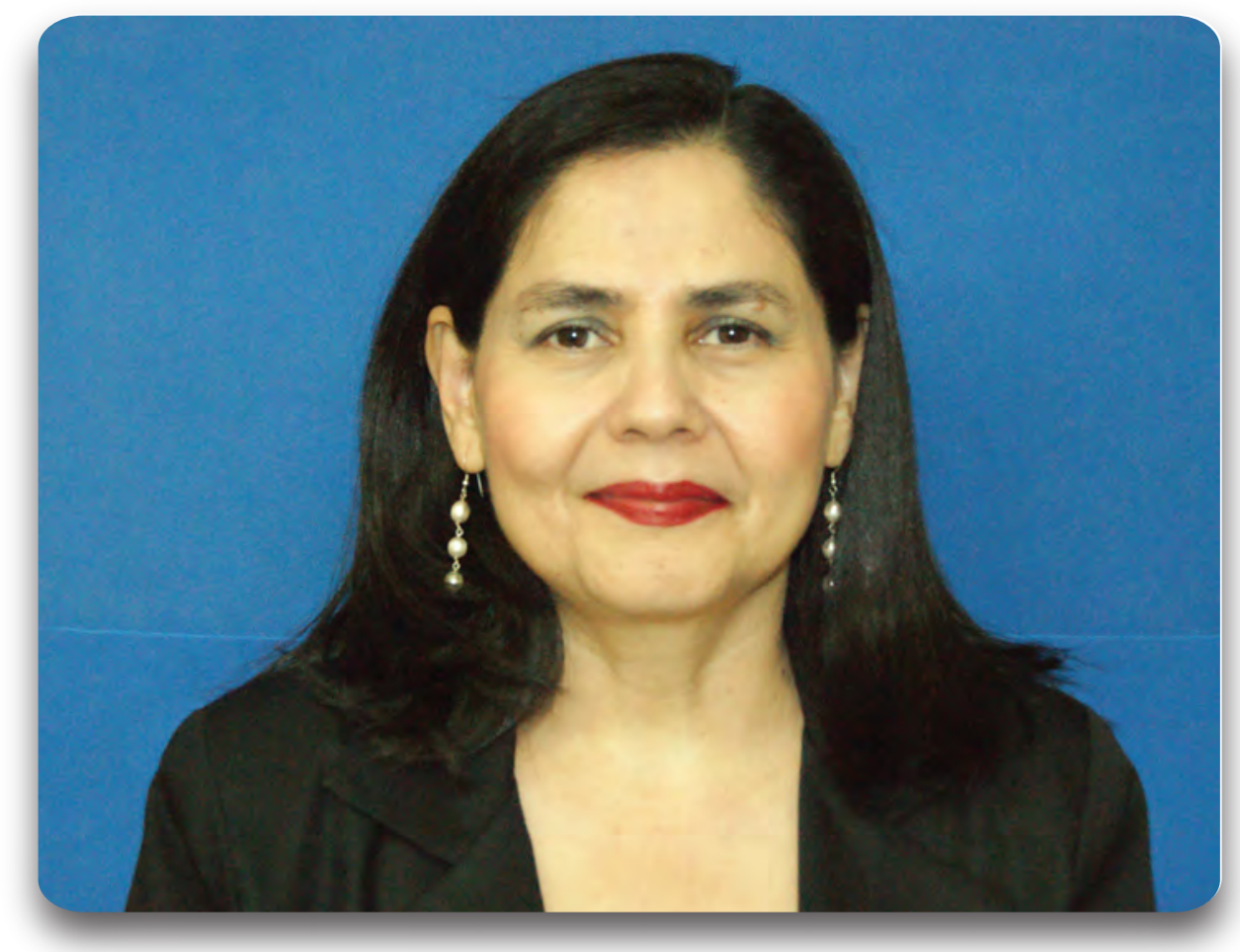

Astarte Alegría Castellanos 